\title{
A Perda Precoce do Diâmetro Luminal após Angioplastia Coronária e sua Influência no Fenômeno da Reestenose
}

\author{
Adriano Mendes Caixeta, Fábio Sândoli de Brito Jr, Pedro Eduardo Horta, M iguel Rati, \\ Mauro Fonseca, Júlia Tizue Fukushima, Siguemituzo A rie, Fúlvio Pileggi
}

São Paulo, SP

\begin{abstract}
Objetivo - Analisar a perda do diâmetro luminal mínimo (DLM) nos primeiros 15 min após angioplastia coronária por balão (AC), quantificando sua influência na reestenose coronária.

Métodos - Foram estudadas, prospectivamente, 86 AC em 86 pacientes. Os pacientes foram divididos em dois $s u b$ grupos de acordo com a presença ou ausência de reestenose; o $1^{o}$ grupo compreendendo as 31 lesões com reestenose e o $2^{\circ}$, as 55 lesões sem reestenose.

Resultados - A análise univariada mostrou que a relação balão/artéria foi menor no grupo com reestenose $(0,92 \pm 0,01$ vs $1,00 \pm 0,11, P=.003)$. O grupo com reestenose apresentou maior recolhimento elástico absoluto e relativo no $1^{\circ} \mathrm{min}(0,79 \pm 0,54$ vs $0,68 \pm 0,59 \mathrm{~mm} ; P=$

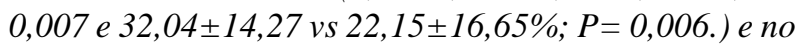
controle angiográfico do $15^{\circ} \mathrm{min}(1,25 \pm 0,59 \mathrm{vs}$ $0,90 \pm 0,65 \mathrm{~mm}, P=0,017$ e 46,75 $\pm 15,69$ vs $29,18 \pm 17,84 \%$, $P<0,00001)$ do que o grupo sem reestenose. O DLM no $1^{\circ}$ min foi menor no grupo com reestenose $(2,15 \pm 0,42 \mathrm{vs}$

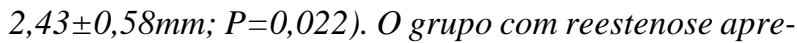
sentou uma maior perda precoce no $\operatorname{DLM}(0,46 \pm 0,34 \mathrm{vs}$ $0,22 \pm 0,35 \mathrm{~mm}, P=0,004)$. Este decréscimo na luz do vaso determinou que o DLM do $15^{\circ} \mathrm{min}$ fosse ainda menor no

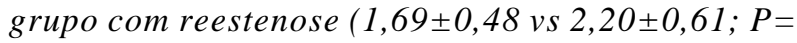
0,0001). Da análise multivariada, entretanto, identificouse apenas a relação balão/artéria e o DLM do $15^{\circ} \mathrm{min}$ como os dois fatores independentes mais relacionados à reestenose.

Conclusão - O recolhimento elástico e a perda do DLM ao longo dos 15 min sãofatores diretamente relacionados à reestenose. Entretanto, a análise multivariada mostrou que a relação balão/artéria e o DLM de $15 \mathrm{~min}$ são os dois fatores independentes mais fortemente preditores de reestenose.
\end{abstract}

\section{Early Luminal Diameter Loss after Percuta- neous Transluminal Coronary Angioplasty and its Relation to the Restenosis Phenomenon}

Purpose - To evaluate the early luminal diameter loss in the first $15 \mathrm{~min}$ after percutaneous transluminal coronary angioplasty (PTCA) and its influence on coronary restenosis.

Methods - In a prospective study, we evaluated 86 patients. The patients were divided in two groups based on the presence or absence of coronary restenosis. Thirty one lesions developed restenosis and 55 lesions did not.

Results - Univariate analysis showed that balloon/ artery ratio was lower in the group of restenosis (0.92 $\pm 0,01$ vs $1.00 \pm 0,11, P=.003)$. Absolute and relative elastic recoil at 1 min was greater in the group that developed restenosis $(0.79 \pm 0.54$ vs $0.68 \pm 0.59 \mathrm{~mm} ; P=.007$ and $32.04 \pm 14.27$ vs $22.15 \pm 16.65 \%$; $P=.006)$. Similarly, absolute and relative elastic recoil at 15 min were greater in the group with restenosis $(1.25 \pm 0.59 \mathrm{vs} 0.90 \pm 0.65 \mathrm{~mm}, P=.017$ e 46.75 \pm 15.69 vs $29.18 \pm 17.84 \%, P<.00001)$. Minimal luminal diameter $(M L D)$ at 1 min was lower in the group with restenosis ( $2.15 \pm 0.42$ vs $2.43 \pm 0.58 \mathrm{~mm} ; P=.022)$. The very early loss was greater in the group with restenosis $(0.46 \pm 0.34$ vs $0.22 \pm 0.35 \mathrm{~mm}, P=.004)$. MLD at 15 min was lower in the group of restenosis than in the group without restenosis (1.69 \pm 0.48 vs $2.20 \pm 0.61 ; P=.0001)$. Multivariate analisys revealed balloon/artery ratio and $M L D$ at 15 min as independent correlates of the late outcome.

Conclusion - The late outcome of PTCA is influenced by elastic recoil and the early MLD loss after PTCA. However, the strongest and most important predictors of late outcome by multivariate analysis were balloon/artery ratio and MLD at $15 \mathrm{~min}$.

Key-words: percutaneous transluminal coronary angioplasty, elastic recoil, restenosis

Arq Bras Cardiol, volume 69 (n 3), 175-179, 1997

Instituto do Coração do Hospital das Clínicas - FMUSP

Correspondência: Adriano Mendes Caixeta - Rua Teodoro Sampaio, 408/101 05406-000 - São Paulo, SP

Recebido para publicação em 4/4/97

Aceito em 16/7/97
O fenômeno da reestenose permanece como a mais importante limitação dos procedimentos de revascularização coronária percutânea ${ }^{1-4}$. Existem, basicamente, três principais mecanismos envolvidos na gênese da reestenose: a proliferação e migração de células musculares lisas, desen- 
cadeando em última análise a hiperplasia intimal ${ }^{5-7}$, o remodelamento geométrico da parede arterial ${ }^{8-10}$ e a perda precoce do diâmetro luminal mínimo (DLM) após a angioplastia $^{11-13}$. O processo de hiperplasia intimal e remodelamento geométrico são mecanismos de reparação à injúria vascular que contribuem para diminuição da luz arterial do vaso a partir de semanas após o procedimento ${ }^{12,13}$. Por outro lado, a perda precoce do diâmetro interno da luz arterial, secundária ao recolhimento elástico, a presença de trombo, dissecção e espasmo, ocorrem a partir de segundos da dilatação com o balão, podendo atuar até nos primeiros dias após a intervenção ${ }^{12,14-17}$. Dentre esses mecanismos, o recolhimento elástico é, sabidamente, o mais freqüentemente envolvido na perda precoce da luz do vaso ${ }^{10,18}$.

Estudos cintilográficos de perfusão miocárdica e testes de esforço realizados entre o $1^{\circ}$ e $3^{\circ}$ dia após angioplastia coronária demonstraram que resultados anormais são preditores de reestenose angiográfica em 6 meses ${ }^{19,20}$. Outros estudos angiográficos associaram o recolhimento elástico imediato ${ }^{11,21} \mathrm{e}$ a perda do DLM em 24 hà presença de reestenose tardia.

O presente estudo objetiva analisar a perda do DLM nos primeiros $15 \mathrm{~min}$ após angioplastia por balão, quantificando a sua influência na reestenose coronária. Além disso, determinar a interferência de outros fatores demográficos, clínicos ou angiográficos mais fortemente relacionados à reestenose, confrontando-os através de testes multivariados.

\section{Métodos}

Foram estudadas, consecutivamente, 86 angioplastias coronárias por cateter balão (AC) em 86 pacientes. Selecionaram-se, prospectivamente, as intervenções realizadas com sucesso angiográfico e clínico (lesão residual $<50 \%$, na ausência de infarto agudo do miocárdio (IAM), cirurgia de emergência, óbito ou necessidade de nova intervenção percutânea ou cirúrgica durante a internação hospitalar). Todos pacientes deste grupo foram submetidos a reestudo angiográfico tardio por critério clínico, funcional ou eletivamente para análise do fenômeno da reestenose, tendo sido incluídos pacientes com angina estável e instável e excluídos os com IAM.

As obstruções coronárias foram classificadas de acordo com a ACC/AHA ${ }^{22}$ modificada por Ellis.

Após acesso femoral ou braquial, realizou-se heparinização sistêmica com dose de 10.000UI a 15.000UI de heparina em bolus e doses adicionais de 5.000UI a cada hora do procedimento. Os pacientes receberam $0,3 \mathrm{mg}$ de nitroglicerina sublingual antes do procedimento e doses de $200 \mu \mathrm{g}$ de nitroprussiato de sódio intracoronária na suspeita de espasmo coronário. A técnica de angioplastia obedeceu aos procedimentos habituais do laboratório de Cardiologia Intervencionista do INCOR ${ }^{23}$.

Os pacientes foram submetidos a estudo angiográfico antes da realização do procedimento, no $1^{\circ} \mathrm{min}$ e no $15^{\circ} \mathrm{min}$ após a última insuflação com sucesso e aos $5 \pm 3$ meses após a AC. O estudo angiográfico da artéria abordada foi realizado na projeção que evidenciasse melhor e o maior grau de severidade da obstrução, sempre na mesma projeção e com as mesmas condições de magnificação radiográfica.

A análise angiográfica quantitativa (AAQ) foi estimada por um único observador, de maneira não duplo-cega, através de caliper eletrônico, tomando-se a ponta do cateter angiográfico para a calibração. Selecionou-se o quadro, no de filme de $35 \mathrm{~mm}$, de melhor contrastação coronária, evidenciando o grau de maior severidade da obstrução e, sempre que possível, no momento tele-diastólico do ciclo cardíaco. Determinaram-se o diâmetro de referência do vaso no segmento arterial livre de doença aterosclerótica proximal à obstrução, o DLM antes do procedimento, no $1^{\circ}$ e $15^{\circ} \mathrm{min}$ após a dilatação com o balão e no reestudo tardio. Quantificaram-se o recolhimento elástico absoluto e relativo e a perda precoce do DLM. O recolhimento elástico absoluto foi definido como a diferença entre o DLM após o procedimento e o diâmetro de máxima insuflação do balão. O recolhimento elástico relativo = recolhimento elástico absoluto $\div$ diâmetro de máxima insuflação do balão x 100. A perda precoce do DLM como a diferença entre os diâmetros luminais mínimos do controle angiográfico do $1^{\circ}$ e do $15^{\circ}$ min.

Os pacientes foram divididos em dois subgrupos de acordo com a presença ou ausência de reestenose coronária no controle angiográfico tardio. Utilizou-se o critério dicotômico para definição de reestenose (obstrução coronária > $50 \%$ à AAQ). $\mathrm{O} 1^{\circ}$ grupo compreendeu as 31 (35\%) lesões que desenvolveram reestenose (com reestenose) e o $2^{\circ}$ grupo as 55 lesões que não apresentaram reestenose (sem reestenose). Compararam-se as variáveis demográficas, clínicas e da análise angiográfica qualitativa e quantitativa entre o grupo com reestenose e o grupo sem reestenose.

As proporções das variáveis categóricas nos dois grupos estudados foram comparadas com o teste do $\mathrm{X}^{2}$ ou teste exato de Fisher. As médias dos dois grupos foram comparadas utilizando-se o teste t de Student. Quando a variável não seguia distribuição normal, os grupos foram comparados com o teste não paramétrico de Wilcoxon. Ajustou-se o modelo de regressão logística com procedimento de seleção stepwise (com o valor de $\mathrm{P}<.25$ da análise univariada utilizado para a seleção). Após testes univariados, aplicou-se a análise multivariada com o intuito de identificar os fatores relacionados, ou mais fortemente relacionados, à reestenose. Considerado significância estatística quando $\mathrm{P}<0,05$

\section{Resultados}

A tabela I mostra as características demográficas, clínicas e angiográficas do grupo sem reestenose e do grupo com reestenose. Não houve diferença entre os dois grupos com relação a idade, sexo, quadro clínico e artéria tratada. No grupo com reestenose predominou as lesões do tipo B2 e C, enquanto que as lesões do tipo A e B1 estiveram mais presentes no grupo sem reestenose. As lesões segmentares 


\begin{tabular}{|c|c|c|c|}
\hline & $\begin{array}{c}\text { Com reestenose } \\
(\mathrm{n}=36)\end{array}$ & $\begin{array}{l}\text { Sem reestenose } \\
\quad(\mathrm{n}=59)\end{array}$ & $\begin{array}{l}\text { Valor de } \\
\quad \mathrm{P}\end{array}$ \\
\hline Idade (anos) & $55 \pm 10$ & $57 \pm 9$ & NS \\
\hline Sexo masculino (\%) & 78 & 7,5 & NS \\
\hline \multicolumn{4}{|l|}{ Quadro clínico (\%) } \\
\hline angina estável & 52 & 56 & NS \\
\hline angina instável & 48 & 44 & NS \\
\hline \multicolumn{4}{|l|}{ Artéria tratada $(\%)$} \\
\hline descendente anterior & 44 & 48 & NS \\
\hline coronária direita & 28 & 26 & NS \\
\hline circunflexa & 28 & 26 & NS \\
\hline Tipo de lesão (\%) & & & $0,002 *$ \\
\hline$A+B 1$ & 29,03 & 63,64 & \\
\hline $\mathrm{B} 2+\mathrm{C}$ & 70,97 & 36,36 & \\
\hline Comprimento (\%) & & & $0,021 *$ \\
\hline$<10 \mathrm{~mm}$ & 61,29 & 83,64 & \\
\hline$>10 \mathrm{~mm}$ & 38,71 & 16,36 & \\
\hline
\end{tabular}

\begin{tabular}{|lccc|}
\hline Tabela II - Análise angiográfica quantitativa comparando o grupo com \\
reestenose e o grupo sem reestenose
\end{tabular}

NS- não significante; DLM- diâmetro luminal mínimo; RE- recolhimento elástico

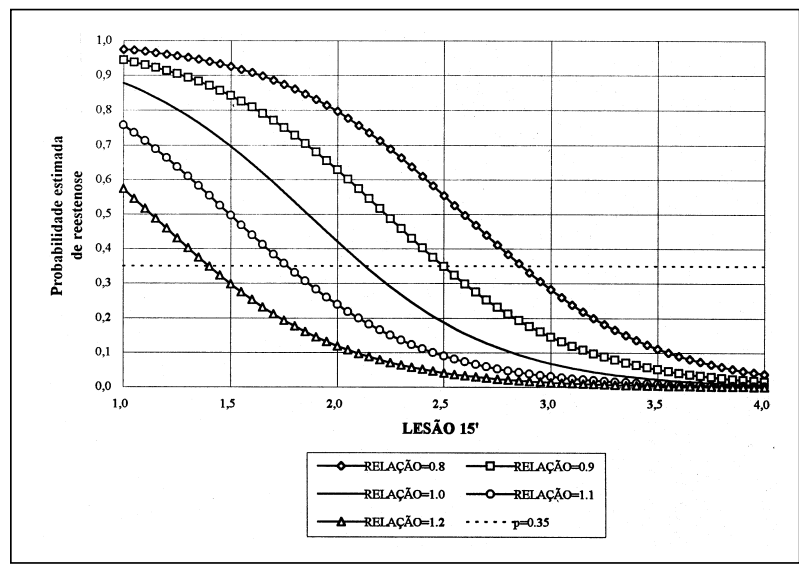

Fig. 1 - Gráfico relacionando a probabilidade de reestenose ao diâmetro luminal mínimo ao final de $15 \mathrm{~min}$ (lesão $15 \mathrm{~min}$ ). As curvas mostram a razão inversa entre a relação balão/artéria e a probabilidade de reestenose.
Perda precoce do diâmetro luminal após ATC e reestenose

(>10mm de comprimento) foi mais freqüente no grupo com reestenose.

Os resultados da AAQ (tab. II) mostram que os diâmetros de referência e os DLM antes do procedimento foram semelhantes nos dois grupos. A relação balão/artéria foi menor no grupo com reestenose $(0,92 \pm 0,01$ vs $1,00 \pm 0,11, \mathrm{P}=$ $0,003)$. O recolhimento elástico absoluto e relativo no $1^{\circ} \mathrm{min}$ foi maior no grupo com reestenose quando comparado ao sem restenose $(0,79 \pm 0,54$ vs $0,68 \pm 0,59 \mathrm{~mm} ; \mathrm{P}=0,007 \mathrm{e}$ $32,04 \pm 14,27$ vs $22,15 \pm 16,65 \%$; $\mathrm{P}=0,006$.). Da mesma maneira, o recolhimento elástico absoluto e relativo no controle angiográfico do $15^{\circ} \mathrm{min}$ foi superior no grupo com reestenose $(1,25 \pm 0,59$ vs $0,90 \pm 0,65 \mathrm{~mm}, \mathrm{P}=0,017 \mathrm{e}$ $46,75 \pm 15,69$ vs $29,18 \pm 17,84 \%, \mathrm{P}<0,00001)$. ODLMnocontrole angiográfico do $1^{\circ} \mathrm{min}$ foi menor no grupo com reestenose $(2,15 \pm 0,42$ vs $2,43 \pm 0,58 \mathrm{~mm} ; \mathrm{P}=0,022)$. O grupo com reestenose apresentou uma perda precoce no DLM estatisticamente maior $(0,46 \pm 0,34 \mathrm{vs} 0,22 \pm 0,35 \mathrm{~mm}, \mathrm{P}=0,004)$. Este decréscimo na luz do vaso determinou que o DLM do controle angiográfico de $15^{\circ} \mathrm{min}$ fosse ainda menor no grupo comreestenose $(1,69 \pm 0,48$ vs $2,20 \pm 0,61 ; P=0,0001)$. A lesãoresidual nos controles angiográficos do $1^{\circ} \mathrm{e}$ do $15^{\circ} \mathrm{min}$ foi, também, maior no grupo com reestenose $(25,30 \pm 0,21$ vs $14,41 \pm 11,12 \%, \mathrm{P}=0,03 \mathrm{e} 41,24 \pm 16,15 \mathrm{vs} 22,14 \pm 11,13 \%, \mathrm{P}=0,015)$.

A partir destes testes univariados, ajustou-se o modelo de regressão logística, objetivando identificar a variável, ou variáveis, mais fortemente associadas à reestenose. As variáveis selecionadas que obedeceram ao critério de inclusão para o teste de regressão logística $(\mathrm{P}<0,25$ da análise univariada) foram: o comprimento da lesão, o tipo de lesão coronária, a relação diâmetro do balão/diâmetro da artéria, o recolhimento elástico absoluto e relativo do $1^{\circ}$ e do $15^{\circ} \mathrm{min}$, a perda precoce do DLM ao longo dos $15 \mathrm{~min}$, o DLM e o percentual de lesão residual no $1^{\circ}$ e do $15^{\circ} \mathrm{min}$. Desta análise multivariada, identificaram-se a relação balão/artéria e o $\mathrm{D} L \mathrm{M}$ do $15^{\circ} \mathrm{min}$ como os dois fatores independentes mais fortemente associados à reestenose.

A partir destes resultados foi possível extrair um modelo estatístico, relacionando a probabilidade de reestenose com a relação balão/artéria e o DLM da angiografia do $15^{\circ}$ min (fig. 1). As obstruções tratadas com uma relação balão/artéria baixa $(<1)$ apresentam maior probabilidade de reestenose que aquelas tratadas com uma relação balão/artéria $>1$. Observa-se, da curva em questão, que o DLM e a probabilidade de reestenose são grandezas inversamente proporcionais. Ou seja, quanto maior o DLM atingido ao final do $15^{\circ} \mathrm{min}$ menor a probabilidade de reestenose coronária, sendo a relação inversa também verdadeira.

\section{Discussão}

Estudos em animais de experimentação e em humanos têm demonstrado a deterioração do diâmetro interno da luz arterial muito precocemente após a angioplastia por cateter balão ${ }^{11-13}$. Esta perda precoce do resultado inicial estaria diretamente relacionada à reestenose angiográfica de 6 meses ${ }^{11,18,21}$. Rozemman e col ${ }^{11}$ demonstraram que o recolhimento elásti- 
co absoluto e relativo no controle angiográfico imediato esteve relacionado ao grupo com reestenose: $0,52 \mathrm{~mm}$ e $18 \%$ nas lesões com reestenose e de $0,37 \mathrm{~mm}$ e $12 \%$ nas lesões sem reestenose. Este estudo mostra, também, que o recolhimento elástico imediato do vaso é fator determinante da lesão residual ao final do procedimento. Em outra publicação, Rodriguez e col $^{24}$ demonstraram que uma perda $>0,3$ mm no DLM ou um incremento de $10 \%$ no diâmetro da obstrução após $24 \mathrm{~h}$ da angioplastia estratificam lesões com maior propensão de desenvolver reestenose no seguimento tardio. Em nossa casuística, similarmente, o recolhimento elástico absoluto e relativo nas angiografias do $1^{\circ}$ e do $15^{\circ} \mathrm{min}$ foram maiores no grupo com reestenose. Da mesma maneira, identificamos que a perda precoce do DLM em $15 \mathrm{~min}$ foi duas vezes maior nas lesões que desenvolveram reestenose $(0,46 \pm 0,34$ vs $0,22 \pm 0,35 \mathrm{~mm}, \mathrm{P}=0,004)$. Estes dados ratificam que o recolhimento elástico e a perda do DLM até o $15^{\circ} \mathrm{min}$ após a dilatação com o balão estão diretamente associados com a reestenose. Entretanto, compilado todos os fatores associados à reestenose através da análise univariada, somente a relação balão/artéria e o DLM do $15^{\circ}$ min emergiram como as duas variáveis independentes preditoras de reestenose. Identificamos que quanto maior a relação balão/artéria menor a probabilidade de reestenose (fig. 1). Este achado converge com os de estudos recentes ${ }^{24,25}$, sugerindo que o aumento na relação balão/artéria interfere favoravelmente no índice de reestenose. Esses autores observaram que uma relação balão/artéria $>0,9$ determina menores lesões residuais ao final do procedimento e, por conseguinte, menor índice de reestenose. É importante ressaltar, entretanto, que a relação balão/artéria atualmente preconizada é de 1:1, a fim de se evitar as dissecções coronárias e suas graves complicações.

A segunda e mais importante variável independente associada à reestenose foi o DLM no $15^{\circ} \mathrm{min}$ após a dilatação com o balão. Este resultado ratifica publicações pregressas ${ }^{26,27}$, demonstrando que o DLM após a angioplastia é a variável angiográfica independente mais fortemente relacionada à reestenose coronária. Em nosso estudo, o diâmetro luminal do $15 \mathrm{~min}$ foi, inclusive, mais fortemente preditor de reestenose que o diâmetro do controle angiográfico imediato.
Assim, todos esses mecanismos de perda precoce da luz arterial (recolhimento elástico, deterioração do DLM nos primeiros minutos após a angioplastia) e a relação balão/artéria são variáveis relacionadas à reestenose que, interagindo, determinam, em última análise o DLM nos $15 \mathrm{~min}$, o mais forte e importante fator independente preditor de reestenose. Neste estudo, o tratamento de obstruções coronárias (relação balão/artéria 1.0) com um DLM $<2,0 \mathrm{~mm}$ ao final do procedimento apresenta probabilidade de reestenose progressivamente $>40 \%$. Por outro lado, diâmetros luminais mínimos $>2,5 \mathrm{~mm}$ apresentaram probabilidade de reestenose estimada $\leq 20 \%$ (fig. 1 ).

Todos esses aspectos ratificam que, além da proliferação intimal e do remodelamento geométrico, mudanças plásticas na parede arterial, que ocorrem muito precocemente após a dilatação com o balão, estariam, também, envolvidos na gênese da reestenose.

Esses resultados sugerem que a dilatação das obstruções coronárias com o balão que atinjam um DLM $>2,5 \mathrm{~mm}$ em $15 \mathrm{~min}$, independente da magnitude do recolhimento elástico e da perda da luz do vaso neste intervalo de tempo, é suficientemente capaz de determinar baixos índices de reestenose. Por outro lado, a despeito de um resultado angiográfico imediato satisfatório que, influenciado pelo recolhimento elástico e pela deterioração da luz interna do vaso, apresente um DLM <2,4mm aos $15 \mathrm{~min}$, a utilização de stents, nessa situação, graças à sua interferência favorável no remodelamento geométrico ${ }^{28}$ e em outros mecanismos associados ao periprocedimento ${ }^{29-32}$, poderia ser uma valiosa arma com o intuito de atingir um maior DLM e, conseqüentemente, melhores resultados angiográficos a longo prazo.

Neste sentido, como sugerido por outros autores ${ }^{33}$, a dilatação com o balão isoladamente, determinando um ótimo resultado angiográfico imediato (provisional stenting: "stent like" balloon angioplasty), poderia desempenhar relevante papel no tratamento percutâneo das obstruções coronárias sem determinar prejuízo nos índices de reestenose. Assim, as indicações eletivas dos stents poderiam ser reservadas às situações de resultados subótimos da angioplastia convencional; ou seja, naquelas condições onde o DLM atingido pelo balão ficasse aquém do oferecido pelo diâmetro arterial.

\section{Referências}

1. Leimgruber PP, Roubin GS, Hollman J et al - Restenosis after successful coronary angioplasty in patients with single vessel disease. Circulation 1986; 73: 71017.

2. Gruentzig AR, King SB III, Schlumpf M, Siegenthaler W- Long-term follow-up after percutaneous transluminal coronary angioplasty: the early Zurich experience. N Engl J Med 1987; 316: 1127-32.

3. Nobuyoshi M, Kimura T, Nosaka H et al - Restenosis after successful percutaneous transluminal coronary angioplasty: serial angiographic follow-up of 229 patients. J Am Coll Cardiol 1988; 12: 616-23.

4. Hishfeld JW Jr, Schwartz JS, Jugo R et al - Restenosis after coronary angioplasty: a multivariate statistical model to relate lesion and procedure variables to restenosis. J Am Coll Cardiol 1991; 18: 647-56.
5. Liu MW, Roubin GS, King SB III - Restenosis after coronary angioplasty. Potential biologic determinants and role of intimal hiperplasia. Circulation 1989; 79: 1374-87.

6. Simons M, Leclerc G, Safian R, Isner J, Weir L, Baim D - Relation between activated smooth-muscle cells in coronary-artery lesions and restenosis after atherectomy. N Engl J Med 1993; 328: 608-13.

7. Macheod DC, Strauss BH, de Jong Met al - Proliferation and extracelular matrix synthesis of smooth muscle cells cultured from human coronary atherosclerosic and restenotic lesion. J Am Coll Cardiol 1994; 23: 59-65.

8. De Franco A, Tuzcu EM, Abdelmegnid A et al - Intravascular ultrasound assessment of PTCA results: insights into the mechanism of balloon angioplasty. J Am Coll Cardiol 1993; 21: 48A. 
9. Mintz GS, Kovach JA, Javier SP, Ditrano CJ, Leon MB - Geometric remodeling is the predominat mechanism of late loss after coronary angioplasty. Circulation 1993; 88: I-654.

10. Post MJ, Borst C, Kuntz R - The relative importance of arterial remodeling compared with intimal hyperplasia in lumen renarrowing after balloon angioplasty. Circulation 1994; 89: 2816-21.

11. Rozenman Y, Gilon D, Welber S, Sapoznikov D, Gotsman MS - Clinical and angiographic predictors of immediate recoil after successful coronary angioplasty and relation to late restenosis. Am J Cardiol 1993; 72: 1020-5.

12. Wilensky RL, March K, Gradus-Pizlo I, Sandusky G, Fineberg N, Hathaway DR - Vascular injury, repair, and restenosis after percutaneous transluminal angioplasty in atherosclerotic rabbit. Circulation 1995; 92: 2995-3005.

13. Waller BF - "Crackers, breakers, stretchers, drillers, scrapers, shavers, burners, welders and melters"- The future treatment of atherosclerotic coronary artery disease? a clinical assessment. J Am Coll Cardiol 1989; 13: 969-87.

14. Hanet C, Wijns W, Michel X, Schroeder E - Influence of balloon size and restenosis morphology on immediate and delayed elastic recoil after precutaneous transluminal coronary angioplasty. J Am Coll Cardiol 1991; 18: 506-11.

15. Rensing BJ, Hermans WR, Beatt KJ et al - Quantitative angiographic assessment of elastic recoil after percutaneous transluminal coronary angioplasty. Am J Cardiol 1990; 66: 1030-44.

16. Uchida Y, Haseagawa K, Kawamura K, Shubuyal I - Angioscopic observation of the coronary luminal changes induced by percutaneous transluminal coronary angioplasty. Am Heart J 1989; 117: 769-76.

17. Honye J, Mahon DJ, Jain A et al - Morphological effects of coronary balloon angioplasty in vivo assessed by intravascular ultrasound imaging. Circulation 1992; 85: 1012-25.

18. Rodríguez AE, Palacios IF, Fernandéz MA, Larribau M, Giraudo M, Ambrose J Time course and mecanism of early luminal diameter loss after percutaneous tranluminal coronary angioplasty. Am J Cardiol 1995; 76: 1131-4.

19. Jain A, Mahmarian JJ, Borges-Neto S et al - Clinical significance of perfusion defects by thallium-201 single photon emission tomography following oral dipyridamolo early after coronary angioplasty. J Am Coll Cardiol 1988; 11: 970-6.

20. El-Tamini H, Davies GJ, Hackett D, Fragasso G, Crea F, Maseri A- Very early prediction of restenosis after successful coronary angioplasty: anatomic and functional assessment. J Am Coll Cardiol 1990; 15: 259-64.

21. Ardissimo D, Di Somma S, Kubica J et al - Influence of elastic recoil on restenosis after successful coronary angioplasty in unstable angina pectoris. Am J Cardiol 1993; 71: 659-63.

22. Ryan TJ, Bauman WB, Kennedy JW et al - Guidelines for percutaneous
Perda precoce do diâmetro luminal após ATC e reestenose

transluminal coronary angioplasty. A report of the American College of Cardiology/American Heart Associacion. Task Force on Assessment of Diagnostic and Therapeutic Cardiovascular Procedures (Committee on Percutaneous Transluminal Coronary Angioplasty). J Am Coll Cardiol 1993; 22: 2033-54.

23. Caixeta AM, Arie S, Brito Jr FS et al - Análise da retração elástica nos primeiros 15 minutos após angioplastia coronária por cateter balão. Arq Bras Cardiol 1996; 66: 5-9.

24. Rodriguez A, Santaera O, Larribeau M, Sosa MI, Palacios I - Early decrease in minimal luminal diameter after successful percutaneous transluminal coronary angioplasty predicts late restenosis. Am J Cardiol 1993; 71: 1391-5.

25. Schmitz HJ, Erbel R, Meyer J, Essen R - Influence of vessel dilatation on restenosis after successful percutaneous transluminal coronary angioplasty. Am Heart J 1996; 131: 884-91.

26. Roubin GS, Douglas JSJ, King SBI et al - Influence of balloon size on initial success, acute complications, and restenosis after percutaneous transluminal coronary angioplasty. A prospective randomized study. Circulation 1988; 78: 557 65 .

27. KuntzRE, Safian RD, Levine MJ, Reis GJ, Diver DJ, Baim DS - Novel approach to analysis of restenosis after the use of three new coronary devices. J Am Coll Cardiol 1992; 19: 1493-9.

28. Mintz GS, Pichard AD, Kent KM et al - Endovascular stents reduce restenosis by eliminating geometric remodeling: a serial intravascular ultrasoud study. J Am Coll Cardiol 1995; 25: 36A.

29. Beatt KJ, Serruys PW, Luijten HE et al - Restenosis after coronary angioplasty: the paradox of increased lumen diameter and restenosis. J Am Coll Cardiol 1992 19: $258-66$

30. Fischman DL, Leon MB, Baim DS et al, for the Stent Restenosis Study Investigators - A randomized comparison of coronary-stent placement and balloon angioplasty in the treatment of coronary artery disease. N Engl J Med 1994; 331 : 496-501.

31. Serruys PW, Jaegere P, Kiemeneij Fet al, for the BENESTENT Study Group - A comparison of balloon-expandable stent implantation with balloon angioplasty in patients with coronary artery disease. N Engl Med 1994; 331: 489-95.

32. Rodriguez AE, Santaera O, Larribau M et al - Coronary stenting decreases restenosis in lesions with early loss in luminal diameter 24 hours after successful PTCA. Circulation 1995; 91: 1397-402.

33. Foley DP, Serruys PW - Provisional stenting: "stent like" balloon angioplasty: Evidence to define the continuing role of balloon angioplasty for percutaneous coronary revascularization. In: Interventional Cardiology and Pharmacology at the Crossroads. Scientific Sessions of American Heart Association, 64 ${ }^{\text {th }}$. New Orleans, 1996: 15-21. 